\title{
Non-Hypoenergetic Graphs with Nullity 2
}

\author{
Saieed Akbari ${ }^{1}$, Somayeh Khalashi Ghezelahmad ${ }^{2, *}$ \\ ${ }^{1}$ Department of Mathematical Sciences, Sharif University of Technology, \\ Tehran, Iran \\ s_akbari@sharif .edu \\ ${ }^{2}$ Department of Mathematics, Science and Research Branch, \\ Islamic Azad University, Tehran, Iran \\ s.ghezelahmad@srbiau.ac.ir
}

(Received March 12, 2021)

\begin{abstract}
The energy of a graph $G$, denoted by $\mathcal{E}(G)$, is defined as the sum of absolute values of all eigenvalues of $G$. A graph of order $n$, whose energy is less than $n$, i.e., $\mathcal{E}(G)<n$, is said to be hypoenergetic. Graphs for which $\mathcal{E}(G) \geq n$ are called non-hypoenergetic. A graph of order $n$ is said to be orderenergetic, if its energy and its order are equal, i.e., $\mathcal{E}(G)=n$. In this paper, we characterize non-hypoenergetic graphs with nullity 2 . It is proved that except two graphs, every connected graph with nullity 2 is non-hypoenergetic.
\end{abstract}

\section{Introduction}

Let $G$ be a simple graph with vertex set $V(G)=\left\{v_{1}, \ldots, v_{n}\right\}$ and edge set $E(G)$. By order and size of $G$, we mean the number of vertices and the number of edges of $G$, respectively. We denote the order of $G$ by $|V(G)|$. For any vertex $v \in V(G)$, the open neighborhood of $v$ in $G$ is $N_{G}(v)=\{u \in V(G): u v \in E(G)\}$. Also the degree of $v$ in $G$ is just $d(v)=\left|N_{G}(v)\right|$. Let $S \subseteq V(G)$. By $\langle S\rangle$, we mean the subgraph of $G$ induced by $S$. The path and the cycle of order $n$ are denoted by $P_{n}$ and $C_{n}$, respectively. A

\footnotetext{
${ }^{*}$ Corresponding author
} 
graph is claw-free, if it has no induced subgraph isomorphic to $K_{1,3}$. A complete bipartite graph with part sizes $m$ and $n$ is denoted by $K_{m, n}$. If $m=n$, then we say that $K_{m, m}$ is balanced. A $\{1,2\}$-subgraph of $G$ is a subgraph which is a disjoint union of a matching and a 2-regular subgraph of $G$. A $\{1,2\}$-subgraph which is a spanning subgraph, is called a $\{1,2\}$-factor.

The adjacency matrix of $G, A(G)=\left[a_{i j}\right]$, is an $n \times n$ matrix, where $a_{i j}=1$ if $v_{i} v_{j} \in E(G)$, and $a_{i j}=0$, otherwise. Thus $A(G)$ is a symmetric matrix and all eigenvalues of $A(G)$ are real. Let $\eta(G)$, the nullity of $G$, denote the number of zero eigenvalues of $A(G)$. The energy of a graph $G, \mathcal{E}(G)$, is defined as the sum of absolute values of eigenvalues of $A(G)$, see [7].

Graphs of order $n$, satisfying the condition $\mathcal{E}(G)<n$ are named hypoenergetic and their properties were studied in [9-11]. Graphs for which $\mathcal{E}(G) \geq n$ are said to be nonhypoenergetic. A graph is called orderenergetic, if its energy and its order are equal, i.e., $\mathcal{E}(G)=n$. Some basic properties of orderenergetic graphs were studied in [3]. The authors showed that there are infinitely many connected orderenergetic graphs. They proved that a graph having a $\{1,2\}$-factor, is orderenergetic if and only if it is a disjoint union of balanced complete bipartite graphs. Also it was established that there is no orderenergetic graph with nullity 1 . The concept of energy was extended by Nikiforov to arbirary complex matrices and digraphs, [13]. In particular, in [2], the authors studied hypoenergetic and non-hypoenergetic digraphs.

In this paper, we investigate some graphs whose energies exceed the number of vertices. We prove that if a graph $G$, has a $\{1,2\}$-subgraph of order $n-1$, then $\mathcal{E}(G)>n$, except for $K_{r, r+1}, r \geq 1$. It is shown that if $K_{2,4}$ is an induced subgraph of a graph $G$ such that $G \backslash V\left(K_{2,4}\right)$ has a perfect matching and $G$ has no component isomorphic to $K_{r, r+2}$, for each $r$, then $\mathcal{E}(G)>n$. It is also proved that if $P_{3}$ is an induced subgraph of a graph $G$, such that $G \backslash V\left(P_{3}\right)$ has a perfect matching and $G$ has no component isomorphic to $K_{r, r+1}$, for each $r$, then $\mathcal{E}(G)>n$. Using these results we show that if $G$ is a claw-free graph of order $n$, then $\mathcal{E}(G)>n$, except for $C_{4}$ and $K_{1,2}$. Furthermore, it is proved that if the nullity of a graph $G$ of order $n$, is 1 , then $\mathcal{E}(G)>n$, except for $K_{1,2}$. Also, we show that except two graphs, every graph with nullity 2 is non-hypoenergetic. In particular, there exist only two connected orderenergetic graphs with nullity 2 . The following lemmas are needed in the sequel. 
Lemma 1. [4] Let $G$ be a graph and $H_{1}, \ldots, H_{k}$ be its $k$ vertex-disjoint induced subgraphs. Then

$$
\mathcal{E}(G) \geq \sum_{i=1}^{k} \mathcal{E}\left(H_{i}\right) .
$$

Lemma 2. [12] Let $H$ be an induced subgraph of a graph $G$. Then $\mathcal{E}(H) \leq \mathcal{E}(G)$ and equality holds if and only if $E(G)=E(H)$,

Lemma 3. [3] Let $G$ be a graph of order $n$. If $G$ has a $\{1,2\}$-factor, then $\mathcal{E}(G) \geq n$. Equality holds if and only if $G$ is a disjoint union of balanced complete bipartite graphs.

Lemma 4. [1,3] If $n$ is an odd integer, then $\mathcal{E}\left(C_{n}\right) \geq n+1$. Moreover, for $n \geq 9$, $\mathcal{E}\left(C_{n}\right) \geq n+2$.

Lemma 5. [3] There is no connected orderenergetic graph with $\eta=1$.

Lemma 6. [12] Let $u$ be a pendent vertex of a graph $G$ and $v$ the vertex in $G$ adjacent to $u$. Then $\eta(G)=\eta(G-u-v)$.

Lemma 7. [5] Let $G$ be a connected claw-free graph. Then it contains a matching which avoids at most one vertex.

\section{Graphs whose energies exceed the number of ver- tices}

In this section, we investigate some graphs whose energies exceed the number of vertices. Furthermore, we show that, except two graphs, every graph with nullity 2 is nonhypoenergetic

Lemma 8. Let $G$ be a graph of order $n$ such that $K_{2,4}$ is an induced subgraph of $G$ and $G \backslash V\left(K_{2,4}\right)$ has a perfect matching. If $G$ has no component isomorphic to $K_{r, r+2}$ (for each $r$ ), then $\mathcal{E}(G)>n$.

Proof. Let $M$ be a perfect matching of $G \backslash V\left(K_{2,4}\right)$. Let $r$ be the maximum number of $P_{2^{-}}$ components of $M$, such that the subgraph induced on $V\left(K_{2,4}\right)$ and these $P_{2}$-components is complete bipartite. With no loss of generality, one can assume that these $P_{2}$-components are $e_{1}, \ldots, e_{r}$. Let $H$ be the subgraph induced on $V\left(K_{2,4}\right)$ and vertices of $e_{1}, \ldots, e_{r}$. (In fact, $H=K_{2+r, 4+r}$.) Since $H$ is not a component of $G$, there exists a $P_{2}$-component of 
$M$, different from $e_{i}, 1 \leq i \leq r$, say $e$, such that $H$ is connected to $e$. We consider two cases:

Case 1. The edge $e$ is connected to $K_{2,4}$. Let $K$ be the subgraph induced on $V\left(K_{2,4}\right)$ and endpoints of $e$. If $K \neq K_{3,5}$, then a computer search shows that $\mathcal{E}(K)>8$. Now, since $G \backslash V(K)$ has a perfect matching, by Lemmas 1 and 3, we find that

$$
\mathcal{E}(G) \geq \mathcal{E}(K)+\mathcal{E}(G \backslash V(K))>8+|V(G \backslash V(K))|>n,
$$

as desired. If $K=K_{3,5}$, then there exists some $e_{j}, 1 \leq j \leq r$, such that the subgraph induced on endpoints of $e$ and $e_{j}$ is not $C_{4}$. Now, let $K^{\prime}$ be the subgraph induced on $V\left(K_{2,4}\right)$ and endpoints of $e$ and $e_{j}$. By a computer search one can find that $\mathcal{E}\left(K^{\prime}\right)>10$. Now, a similar argument as above implies that $\mathcal{E}(G)>n$.

Case 2. The edge $e$ is connected to some $e_{i}, 1 \leq i \leq r$ and it is not connected to $K_{2,4}$. Let $K^{\prime \prime}$ be the subgraph induced on $V\left(K_{2,4}\right)$ and endpoints of $e$ and $e_{i}$. A computer search shows that $\mathcal{E}\left(K^{\prime \prime}\right)>10$. Therefore as we did before $\mathcal{E}(G)>n$. This completes the proof.

Lemma 9. Let $G$ be a graph of order $n$ such that $P_{3}$ is an induced subgraph of $G$ and $G \backslash V\left(P_{3}\right)$ has a perfect matching. If $G$ has no component isomorphic to $K_{r, r+1}$ (for each $r)$, then $\mathcal{E}(G)>n$.

Proof. Let $M$ be a perfect matching of $G \backslash V\left(P_{3}\right)$. Let $e_{1}, \ldots, e_{r}, r \geq 1$ be the maximum number of $P_{2}$-components of $M$, such that the subgraph induced on $V\left(P_{3}\right)$ and the vertices of these $P_{2}$-components is complete bipartite. Let $H$ be the subgraph induced on $V\left(P_{3}\right)$ and vertices of $e_{1}, \ldots, e_{r}$. (In fact, $H=K_{r+1, r+2}$.) Since $H$ is not a component of $G$, there exists a $P_{2}$-components of $M$, different from $e_{i}, 1 \leq i \leq r$, say $e$, such that $H$ is connected to $e$. We consider two cases:

Case 1. The edge $e$ is connected to $P_{3}$. Let $K$ be the subgraph induced on $V\left(P_{3}\right)$ and endpoints of $e$. If $K \neq K_{2,3}$, then a computer search shows that $\mathcal{E}(K) \geq 5.22$. Now, since $G \backslash V(K)$ has a perfect matching, by Lemmas 1 and 3, we find that

$$
\mathcal{E}(G) \geq \mathcal{E}(K)+\mathcal{E}(G \backslash V(K))>5+|V(G \backslash V(K))|>n,
$$

as desired. If $K=K_{2,3}$, then there exists some $e_{j}, 1 \leq j \leq r$, such that the subgraph induced on endpoints of $e$ and $e_{j}$ is not $C_{4}$. Now, let $K^{\prime}$ be the subgraph induced on 
$V\left(P_{3}\right)$ and endpoints of $e$ and $e_{j}$. By a computer search one can find that $\mathcal{E}\left(K^{\prime}\right) \geq 7.94$. Now, a similar argument as above implies that $\mathcal{E}(G)>n$.

Case 2. The edge $e$ is connected to some $e_{i}, 1 \leq i \leq r$ and it is not connected to $P_{3}$. Let $K^{\prime \prime}$ be the subgraph induced on $V\left(P_{3}\right)$ and end points of $e$ and $e_{i}$. A computer search shows that $\mathcal{E}\left(K^{\prime \prime}\right)>7.45$. Hence as we did before $\mathcal{E}(G)>n$.

Lemma 10. Let $G$ be a graph of order $n$ such that $2 P_{3}=P_{3} \cup P_{3}$ is an induced subgraph of $G$ and $G \backslash V\left(2 P_{3}\right)$ has a perfect matching. If $G$ has no component isomorphic to $K_{r, r+1}$ (for each $r$ ), then $\mathcal{E}(G)>n$.

Proof. Assume that $W_{1}$ and $W_{2}$ are $P_{3}$-components of $G$. Let $M$ be a perfect matching of $G \backslash V\left(W_{1} \cup W_{2}\right)$. Let $e_{1}, \ldots, e_{r}, r \geq 1$ be the maximum number of $P_{2}$-components of $M$, such that the subgraph $H$ induced on $V\left(W_{1}\right)$ and vertices of $e_{1}, \ldots, e_{r}$ is complete bipartite. Since $H$ is not a component of $G$, either $H$ is connected to some $P_{2}$-component of $M$, say $e$, where $e \neq e_{i}, 1 \leq i \leq r$, or $H$ is connected to $W_{2}$. Two cases can be considered:

Case 1. Assume that the subgraph $H$ is connected to some $P_{2}$-component $e$ of $M$, where $e$ is different from $e_{i}, 1 \leq i \leq r$. Then the argument used in the proof of Lemma 9 , implies that $W_{1}$ is contained in a connected subgraph $K$ of $G$, where $K$ is of order 5 or 7 and $\mathcal{E}(K)>|V(K)|$. As we stated in the proof of Lemma 9, if $K$ is of order 5 , then $\mathcal{E}(K) \geq 5.22$ and if $K$ is of order 7 , then $\mathcal{E}(K) \geq 7.45$. First, suppose that $K$ is of order 5. Now, since $\mathcal{E}\left(W_{2}\right)=2.82$ and $G \backslash\left[V(K) \cup V\left(W_{2}\right)\right]$ has a perfect matching, we obtain

$$
\begin{gathered}
\mathcal{E}(G) \geq \mathcal{E}(K)+\mathcal{E}\left(W_{2}\right)+\mathcal{E}\left(G \backslash\left[V(K) \cup V\left(W_{2}\right)\right]\right) \geq \\
5.22+2.82+\left|V\left(G \backslash\left[V(K) \cup V\left(W_{2}\right)\right]\right)\right|>n .
\end{gathered}
$$

Next, assume that the order of $K$ is 7 . We have

$$
\begin{gathered}
\mathcal{E}(G) \geq \mathcal{E}(K)+\mathcal{E}\left(W_{2}\right)+\mathcal{E}\left(G \backslash\left[V(K) \cup V\left(W_{2}\right)\right]\right) \geq \\
7.45+2.82+\left|V\left(G \backslash\left[V(K) \cup V\left(W_{2}\right)\right]\right)\right|>n,
\end{gathered}
$$

as desired. 
Case 2. Assume that the subgraph $H$ is connected to $W_{2}$. Then there exists some $e_{j}, 1 \leq j \leq r$, such that $W_{2}$ is connected to $e_{j}$. Let $K^{\prime}$ be the subgraph induced on $V\left(W_{1}\right) \cup V\left(W_{2}\right)$ and endpoints of $e_{j}$. A computer search shows that $\mathcal{E}\left(K^{\prime}\right)>8$. Now, since $G \backslash V\left(K^{\prime}\right)$ has a perfect matching, we find that $\mathcal{E}(G)>n$. This completes the proof.

Corollary 11. Let $G$ be a connected graph of order $n$. If $G$ has a $\{1,2\}$-subgraph of order $n-1$, then $\mathcal{E}(G)>n$, except for $G=K_{r, r+1}, r \geq 1$. In particular, if the nullity of $G$ is 1 , then $\mathcal{E}(G)>n$, except for $K_{1,2}$.

Proof. Let $H$ be a $\{1,2\}$-subgraph of order $n-1$. Let $V(G) \backslash V(H)=\{u\}$. First assume that $H$ contains at least one odd cycle, say $C$. One may assume that every odd cycle in $H$ is an induced odd cycle, because if we have an odd cycle with a chord, then there is a chord which partitions the vertices of odd cycle into an induced odd cycle and some paths of order 2. Furthermore, we can assume that $H$ has no even cycle, because the vertex set of every even cycle can be partitioned into disjoint copies of $P_{2}$. Now, since $\langle V(H)\rangle$ is a proper subgraph of $G$, by Lemmas 1, 2 and 4 we obtain

$\mathcal{E}(G)>\mathcal{E}(\langle V(H)\rangle) \geq \mathcal{E}(C)+\mathcal{E}(\langle V(H) \backslash V(C)\rangle) \geq(|V(C)|+1)+|\langle V(H) \backslash V(C)\rangle| \geq n$

so we are done.

Next, suppose that every component of $H$ is $P_{2}$. Let $v w$ be the $P_{2}$-component of $H$, such that $v \in N(u)$. Let $K=\langle u, v, w\rangle$. If $K=C_{3}$, then $G$ has a $\{1,2\}$-factor. Hence, by Lemma $3, \mathcal{E}(G) \geq n$. Note, that in the equality case, $G$ should a balanced complete bipartite graph, but this contradicts the existence of $C_{3}$ in $G$. Thus $\mathcal{E}(G)>n$. If $K=P_{3}$, then Lemma 9, implies that $\mathcal{E}(G)>n$, as desired. In order to prove the last assertion, let $\phi(G, x)=\sum_{i=0}^{n} c_{i} x^{n-i}$ be the characteristic polynomial of $G$. Since $\eta(G)=1, c_{n-1} \neq 0$. Now, by Sachs Theorem [12, p. 7], $G$ contains a $\{1,2\}$-subgraph of order $n-1$. Hence the result follows from the previous part.

Remark 12. Note that Corollary 11, implies Lemma 5.

Corollary 13. If $G$ is a connected claw-free graph of order $n$, then $\mathcal{E}(G)>n$, except for $K_{1,2}$ and $C_{4}$. 
Proof. Let $G$ be a claw-free graph of order $n$. Then by Lemma 7 , either $G$ has a $\{1,2\}$ factor, or it has a $\{1,2\}$-subgraph of order $n-1$. First, assume that $G$ has a $\{1,2\}$-factor, then by Lemma $3, \mathcal{E}(G) \geq n$. If the equality holds, then $G$ is a balanced complete bipartite graph. However, since $G$ is claw-free, this is only possible if $G=C_{4}$. Hence $\mathcal{E}(G)>n$, except for $C_{4}$. Next, suppose that $G$ has a $\{1,2\}$-subgraph of order $n-1$. Then by Corollary $11, \mathcal{E}(G)>n$, except for $K_{1,2}$. So we are done.

Now, we are in a position to state the main theorem of this section.

Theorem 14. Let $G$ be a connected graph of order $n$ with nullity 2 . If $G \notin\left\{K_{1,3}, C_{4}, G_{1}, G_{2}\right\}$, then $\mathcal{E}(G)>n$. In particular, $C_{4}$ and Graph $G_{1}$ are the only orderenergetic graphs with nullity 2.

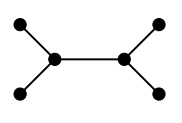

$G_{1}$

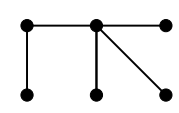

$G_{2}$

Proof. Let $\phi(G, x)=\sum_{i=0}^{n} c_{i} x^{n-i}$ be the characteristic polynomial of $G$. Since $\eta(G)=2$, $c_{n-2} \neq 0$. Now, by Sachs Theorem, $G$ contains a $\{1,2\}$-subgraph of order $n-2$, say $H$. Let $V(G) \backslash V(H)=\{u, v\}$. Note that if $u$ and $v$ are adjacent, then $G$ has a $\{1,2\}$-factor. Hence by Lemma $3, \mathcal{E}(G) \geq n$. However, if $\mathcal{E}(G)=n$, then $G$ is a balanced complete bipartite graph. But the only balanced complete bipartite graph with nullity 2 is $C_{4}$, which contradicts the assumption. Therefore $\mathcal{E}(G)>n$. Hence in the following we may assume that $u$ and $v$ are not adjacent.

Case 1. Assume that $H$ contains at least one induced odd cycle. Let $C$ be a cyclecomponent of $H$. First, suppose that at least one of the $u$ or $v$ is adjacent to $C$. Then it is easy to see that $G$ has a $\{1,2\}$-subgraph of order $n-1$. Now, by Corollary $11, \mathcal{E}(G)>n$. (Note that since $\eta=2, G$ is not isomorphic to $K_{r, r+1}$, for some $r$.) Next assume that both $u$ and $v$ are adjacent to the same $P_{2}$ component of $H$, say $x y$. Let $K=\langle u, v, x, y\rangle$. Note that if $K$ has a perfect matching, then $G$ has a $\{1,2\}$-factor and a similar augment as above yields that $\mathcal{E}(G)>n$. So we may exclude subgraphs $K$ with a perfect matching. It follows that $K=K_{1,3}$. Now, since $G \backslash(V(K) \cup V(C))$ has a perfect matching and $\mathcal{E}(K)=3.46$, using Lemmas 1, 3 and 4 we have:

$$
\mathcal{E}(G) \geq \mathcal{E}(K)+\mathcal{E}(C)+\mathcal{E}(G \backslash[V(K) \cup V(C)]) \geq
$$




$$
(|V(K)|-0.54)+(|V(C)|+1)+\mid V(G \backslash[V(K) \cup V(C)] \mid>n .
$$

Finally, suppose that $u$ and $v$ are adjacent to different $P_{2}$-components of $H$, say $w z$ and $r s$, respectively. Let $u \in N(w)$ and $v \in N(r)$. Let $N_{1}=\langle u, w, z\rangle$ and $N_{2}=\langle v, r, s\rangle$. We note that if $N_{1}$ or $N_{2}$ is $C_{3}$, then $G$ has a $\{1,2\}$-subgraph of order $n-1$. Hence by Corollary $11, \mathcal{E}(G)>n$. So we let $N_{1}=N_{2}=P_{3}$. We have $\mathcal{E}\left(P_{3}\right)=2.82$. Now, by Lemmas 1, 3 and 4 we obtain:

$$
\begin{gathered}
\mathcal{E}(G) \geq \mathcal{E}(C)+\mathcal{E}\left(N_{1}\right)+\mathcal{E}\left(N_{2}\right)+\mathcal{E}\left(G \backslash\left[V(C) \cup V\left(N_{1}\right) \cup V\left(N_{2}\right)\right]\right) \geq \\
(|V(C)|+1)+\left(\left|V\left(N_{1}\right)\right|-0.18\right)+\left(\left|V\left(N_{2}\right)\right|-0.18\right)+\left|V\left(G \backslash\left[V(C) \cup V\left(N_{1}\right) \cup V\left(N_{2}\right)\right]\right)\right|>n,
\end{gathered}
$$
as desired.

Case 2. Assume that every component of $H$ is $P_{2}$. Note that this is only possible if $n$ is even. We consider three cases:

Subcase 2.1. If $u$ and $v$ are adjacent to different vertices of a $P_{2}$-component of $H$, then $G$ has a $\{1,2\}$-factor. So $\mathcal{E}(G)>n$.

Subcase 2.2. If both $u$ and $v$ are adjacent to one vertex of a $P_{2}$-component of $H$, say $x y$, then let $K=\langle u, v, x, y\rangle$. We have $K=K_{1,3}$. Let $x$ be the central vertex of $K=K_{1,3}$ and $L=G \backslash V(K)$. Clearly, $L$ has a perfect matching, say $M$. Two cases can be considered:

(a) Assume that $u, v$ and $y$ are pendent vertices of $G$. First note that, by Lemma 6 , $\eta(G-u-x)=2$. Clearly, $G-u-x=2 K_{1} \cup L$. Hence $\eta(L)=0$. Now, since $G$ is connected, there exists a $P_{2}$-component of $M$, say $a b$, such that $a \in N(x)$. Let $W=\langle a, b, u, v, x, y\rangle$. A computer search shows that if $W$ is not Graph $(a)$, then $\mathcal{E}(W)>6$. Note that the nullity of Graph $(a)$ is 2 and its energy is 5.81 .

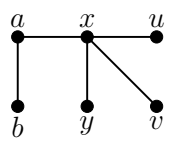

(a) 
So if $W$ is not Graph $(a)$, then since $G \backslash V(W)$ has a perfect matching, we obtain

$$
\mathcal{E}(G) \geq \mathcal{E}(W)+\mathcal{E}(G \backslash V(W))>6+(n-6)>n
$$

If $W$ is Graph $(a)$, then there exists a $P_{2}$-component of $M$, say $c d$ such that $c$ or $d$ is adjacent to some vertex of $W$ different from $u, v$ and $y$. Let $W^{\prime}=\langle V(W), c, d\rangle$. We observed that except Graphs $(b)$ and $(c), \mathcal{E}\left(W^{\prime}\right)>8$. This implies that $\mathcal{E}(G)>n$.

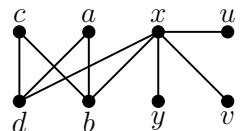

(b)

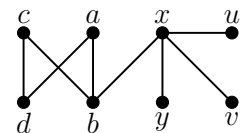

(c)

If $W^{\prime}$ is one of the Graphs $(b)$ or $(c)$, then we note that $C_{4}$ is a subgraph of $L$. But since $\eta(L)=0, C_{4}$ is not a component of $L$. Let $N$ be the component of $L$ which contains $C_{4}$. Clearly, $N$ is not balanced complete bipartite, otherwise $\eta(L) \geq 2$. Moreover, $N$ has a perfect matching, say $M_{1}$. Let $M^{\prime}$ be a proper subset of $M_{1}$, such that the subgraph $N^{\prime}=\left\langle V\left(M^{\prime}\right)\right\rangle$ is the balanced complete bipartite subgraph of $N$ of maximum order. Since $C_{4}$ is a subgraph of $N$, the order of $N^{\prime}$ is at least 4 . Now, since $N$ is connected, there exists a $P_{2}$-component of $M_{1} \backslash M^{\prime}$, say $r s$, such that $N^{\prime}$ is connected to $r s$. This means that there is a $P_{2}$-component of $M^{\prime}$, say ef such that the subgraph $\langle e, f, r, s\rangle$ is connected. Now, if $\langle e, f, r, s\rangle \neq K_{2,2}$, then let $g h \in M^{\prime} \backslash\{e f\}$ be arbitrary and consider $N_{1}=\langle e, f, g, h, r, s\rangle$ (Note that since the order of $N^{\prime}$ is at least 4 , the edge $g h$ exists). A computer search shows that $\mathcal{E}\left(N_{1}\right) \geq 6$.63. If $\langle e, f, r, s\rangle=K_{2,2}$, then let $g h \in M^{\prime} \backslash\{e f\}$ such that $\langle g, h, r, s\rangle \neq K_{2,2}$. Note that $g h$ exists, otherwise $\left\langle V\left(N^{\prime}\right), r, s\right\rangle$ is a balanced complete bipartite subgraph of $N$ whose order is greater than $N^{\prime}$, a contradiction. Now, consider the subgraph $N_{2}=\langle e, f, g, h, r, s\rangle$. We observed that $\mathcal{E}\left(N_{2}\right) \geq 6.92$. Thus for $i=1,2$ we have

$$
\mathcal{E}(G) \geq \mathcal{E}(K)+\mathcal{E}\left(N_{i}\right)+\mathcal{E}\left(G \backslash\left[V(K) \cup V\left(N_{i}\right)\right]\right) .
$$

Since $G \backslash\left[V(K) \cup V\left(N_{i}\right)\right]$ has a perfect matching, we obtain

$$
\mathcal{E}(G) \geq 3.46+6.63+\left|V\left(G \backslash\left[V(K) \cup V\left(N_{i}\right)\right]\right)\right|>n .
$$


(b) Suppose that at least one of the vertices $u, v$ and $y$ is not a pendent vertex of $G$, say $u$. We remind that the subgraph $K=\langle u, v, x, y\rangle$ is $K_{1,3}$ and $L=G \backslash V(K)$ has a perfect matching, $M$. Now, since $d(u)>1$ and $v, y \notin N(u)$, there exists a $P_{2}$-component $w z$ of $M$ such that $w \in N(u)$. Let $W=\langle u, v, x, y, w, z\rangle$. Again we exclude subgraphs $W$ with a perfect matching. Now, a computer search shows that except $W=K_{2,4}, \mathcal{E}(W)>6$, which implies that $\mathcal{E}(G)>n$. So assume that $W=K_{2,4}$. Clearly, $G \backslash V(W)$ has a perfect matching. Now, Lemma 8 , yields that $\mathcal{E}(G)>n$.

Subcase 2.3. Suppose that there exist $P_{2}$-components $x y$ and $w z$ of $H$, such that $x \in N(u)$ and $w \in N(v)$. Let $W=\langle u, v, x, y, w, z\rangle$. As we discussed in the previous subcase, we can exclude subgraphs $W$ with a perfect matching. Now, a computer search shows that except $2 P_{3}, K_{2,4}$ and the Graph $(d), \mathcal{E}(W)>6$, which implies that $\mathcal{E}(G)>n$ :

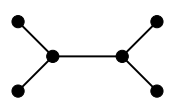

$(d)$

Note that Graph $(d)$, is orderenergetic and has nullity 2. Assume that $n>6$. We consider three cases:

(a) If $W$ is Graph $(d)$, then since $G$ is connected, there exists a $P_{2}$-component of $H \backslash V(W)$, say $r s$, such that $r$ or $s$ is adjacent to some vertex of $W$. Now, consider the subgraph $\langle V(W), r, s\rangle$. A computer search shows that the energy of this subgraph is greater that 8 . Consequently, $\mathcal{E}(G)>n$.

(b) If $W=K_{2,4}$, then by Lemma 8 we find that $\mathcal{E}(G)>n$.

(c) If $W=2 P_{3}$, then Lemma 10 implies that $\mathcal{E}(G)>n$. The proof is complete.

Acknowledgments: The research of the first author was supported by grant number (G981202) from the Sharif University of Technology. 


\section{References}

[1] A. Aashtab, S. Akbari, E. Ghasemian, A. H. Ghodrati, M. A. Hosseinzadeh, F. Koorepazan-Moftakhar, On the minimum energy of regular graphs, Lin. Algebra Appl. 581 (2019) 51-71.

[2] S. Akbari, K. C. Das, S. Khalashi Ghezelahmad, F. Koorepazan-Moftakhar, Hypoenergetic and nonhypoenergetic digraphs, Lin. Algebra Appl. 618 (2021) 129-143.

[3] S. Akbari, M. Ghahremani, I. Gutman, F. Koorepazan-Moftakhar, Orderenergetic graphs, MATCH Commun. Math. Comput. Chem. 84 (2020) 325-334.

[4] S. Akbari, E. Ghorbani, M. R. Oboudi, Edge addition, singular values, and energy of graphs and matrices, Lin. Algebra Appl. 430 (2009) 2192-2199.

[5] J. Akiyama, M. Kano, Factors and Factorizations of Graphs - Proof Techniques in Factor Theory, Springer, Berlin, 2011.

[6] R. B. Bapat, S. Pati, Energy of a graph is never an odd integer, Bull. Kerala Math. Assoc. 1 (2004) 129-132.

[7] I. Gutman, The energy of a graph, Ber. Math. Statist. Sekt. Forschungsz. Graz. 103 (1978) 1-22.

[8] I. Gutman, S. Radenković, Hypoenergetic molecular graphs, Indian J. Chem. 46A (2007) 1733-1736.

[9] I. Gutman, Hyperenergetic and hypoenergetic graphs, in: D. Cvetković, I. Gutman (Eds.), Selected Topics on Applications of Graph Spectra, Math. Inst., Belgrade, 2011, $113-135$.

[10] I. Gutman, X. Li, Y. Shi, J. Zhang, Hypoenergetic trees, MATCH Commun. Math. Comput. Chem. 60 (2008) 415-426.

[11] I. Gutman, S. Radenković, Hypoenergetic molecular graphs, Indian J. Chem. 46A (2007) 1733-1736.

[12] X. Li, Y. Shi, I. Gutman, Graph Energy, Springer, New York, 2012.

[13] V. Nikiforov, The energy of graphs and matrices, J. Math. Anal. Appl. 326 (2007) 1472-1475. 